Brit. Heart F., 1967, 29, 520.

\title{
Intermittent Complete Left Bundle-branch Block: Phonocardiographic and Mechanocardiographic Study of One Case
}

\author{
J. BARAGAN, F. FERNANDEZ-CAAMANO, B. COBLENCE, AND J. LENÈGRE \\ From the Clinique Cardiologique de l'Hôpital Boucicaut, Paris 15e, France
}

Since the work of Wolferth and Margolies (1935), the modifications of both the heart sounds and the sequence of ventricular events induced by left bundle-branch block have been well known. Yet there has been some disagreement on details, mainly because left bundle-branch block is seldom an isolated abnormality. The opportunity was offered to record the heart sounds, the indirect carotid pulse, and the apex cardiogram in the same patient, in conditions of both normal and abnormal left intraventricular conduction, making it possible to describe the changes due to the complete bundlebranch block alone.

\section{METHOD}

The electrocardiogram, phonocardiogram, indirect carotid pulse curve, and the apex cardiogram were recorded with a photographic 4-channel Hellige Multicardiotest. The phonocardiogram, dissociated into three frequency bands, low, medium, and high, ranging respectively from 5 to 50,25 to 150 , and 50 to 250 cycles per second, was taken successively from three areas, apical, pulmonary, and aortic, simultaneously with the electrocardiographic lead I. Next, these same phonocardiograms (low and high frequencies only) and electrocardiographic lead I were recorded either with an indirect carotid pulse curve (capacitance transducer Infraton-E system) or with an apex cardiogram (piezoelectric crystal transducer). Paper speed was $50 \mathrm{~mm}$. per second with $0.02 \mathrm{sec}$. intervals between the vertical lines.

The following time intervals were measured after Tafur, Cohen, and Levine, 1964 (Fig. 1).

(1) Electromechanical interval (EMI) from the onset

Received July 11, 1966. of the QRS complex of the electrocardiogram to the onset of the systolic wave of the apex cardiogram (ACG).

(2) First pre-ejection component of ventricular contraction (PEC-I) from the onset of the systolic wave of the ACG to the notch on its ascending limb which marks mitral valve closure (MC), and corresponds normally to the first rapid vibrations of the 1 st heart sound.

(3) Second pre-ejection component of ventricular contraction (PEC-II) from MC on the ascending limb of the ACG to the onset of the indirect carotid pulse (OCP). In the present study, this interval was not measured but calculated by subtraction of the average $Q R S$ to $M C$ interval from the average $Q R S$ to $O C P$ interval (Q-OCP).

(4) Ejection phase of ventricular contraction from the OCP to its dicrotic notch, which marks aortic valve closure.

(5) Isometric relaxation phase from the aortic component of the 2nd sound (A2) on the phonocardiogram to the lower-most point " $O$ " of the ACG, which marks mitral valve opening.

(6) Ventricular filling phase from the point " $O$ " to the onset of the following systolic wave of the ACG.

Each one of these intervals was measured in 10 different complexes, corrected for heart rate according to Bazett's (1920) formula (corrected interval = measured interval divided by the square root of the preceding $\mathbf{R} R$ interval of the electrocardiogram). The average of the corrected values was taken. Routine statistical analysis was carried out on these corrected values, and Student's " $t$ " test used to express the significance of the difference between the measurements with or without bundlebranch block.

\section{CASe Report}

A woman aged 55 attended hospital complaining of vague, irregular pains in the left chest unrelated to effort. No relevant past history was found except for mild transient hypertension, 10 years previously. On 520 

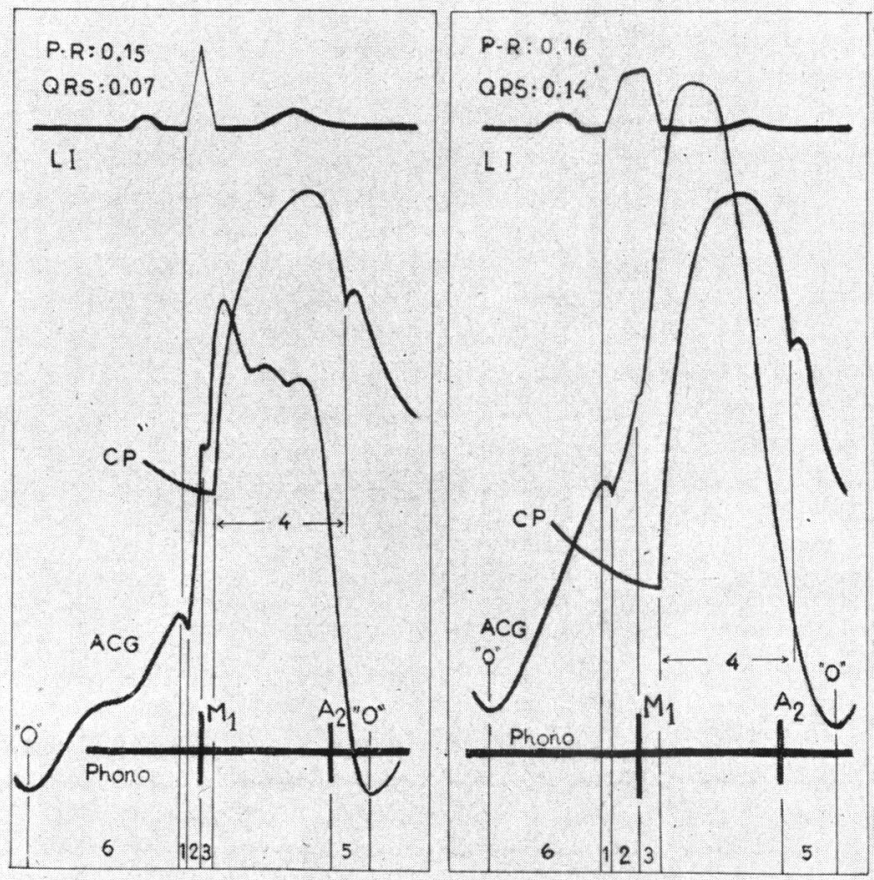

FIG. 1.-Diagram of the various phases of left ventricular contraction in incomplete (left side) and complete (right side) left bundle-branch block. From above downwards: electrocardiographic lead I, carotid pulse (CP), apex cardiogram (ACG) "O": mitral opening, phonocardiogram (M1: mitral component of the 1st sound, A2: aortic component of the 2nd sound). 1: Electromechanical interval, 2 and 3: respectively first and second pre-ejection components of ventricular contraction, 4: ejection phase, 5: isometric relaxation phase, $6+1$ : ventricular filling phase. Note in complete block, a normal electromechanical interval (1), prolongation of both pre-ejection components of ventricular contraction ( 2 and 3 ), and isometric relaxation phase (5), shortened ventricular filling phase $(6+1)$.

mm. Hg. The heart was considered normal on auscultation, in spite of a $1 / 6$ systolic murmur in the aortic area, and also on $x$-ray examination. The resting electrocardiogram was practically normal, with perhaps a mild degree of incomplete left bundle-branch block in view of absent $q$ waves in lead $I$ and of an $r^{\prime} R^{\prime}$ pattern in leads V5-V6: P-R interval $0.15 \mathrm{sec}$., QRS interval 0.07 sec., ventricular activation time in V6 0.05 sec., normal repolarization waves. Simple acceleration of the heart rate from 76 to 80 a minute, induced transiently by a single cough, resulted in a typical pattern of "complete left bundle-branch block": P-R interval 0.16 sec., QRS interval $0 \cdot 13$ to $0.14 \mathrm{sec}$, delayed ventricular activation time to $0.09 \mathrm{sec}$. in V6, downward displacement of the ST segment, with negative $T$ waves in leads V6 and V7 (Fig. 2).

\section{RESULTS}

In this case, the onset of an electrical pattern of complete left bundle-branch block resulted in the following modifications.
Phonocardiogram (Fig. 3). (1) Diminished amplitude and increased duration (from 0.16 to $0.20 \mathrm{sec}$.) of the first heart sound, which was then formed by three discrete waves. (2) Splitting of the second heart sound, with an interval of $0.06 \mathrm{sec}$. between its two components.

Indirect carotid pulse curve (Fig. 1 and 4 and Table). (1) Delayed onset, by $0.067 \mathrm{sec}$., of the systolic wave, which corresponded with the third wave of the widened first heart sound (aortic valve opening). (2) Slight shortening, by $0.027 \mathrm{sec}$., of systolic ejection time (not significant). (3) Occurrence of both components of the second heart sound before the dicrotic notch (reversed splitting).

Apex cardiogram (Fig. 1 and 5 and Table). (1) Slight increase, by 0.005 sec., of the EMI (not significant); (2) conspicuously increased duration of both pre-ejection components, PEC-I and 

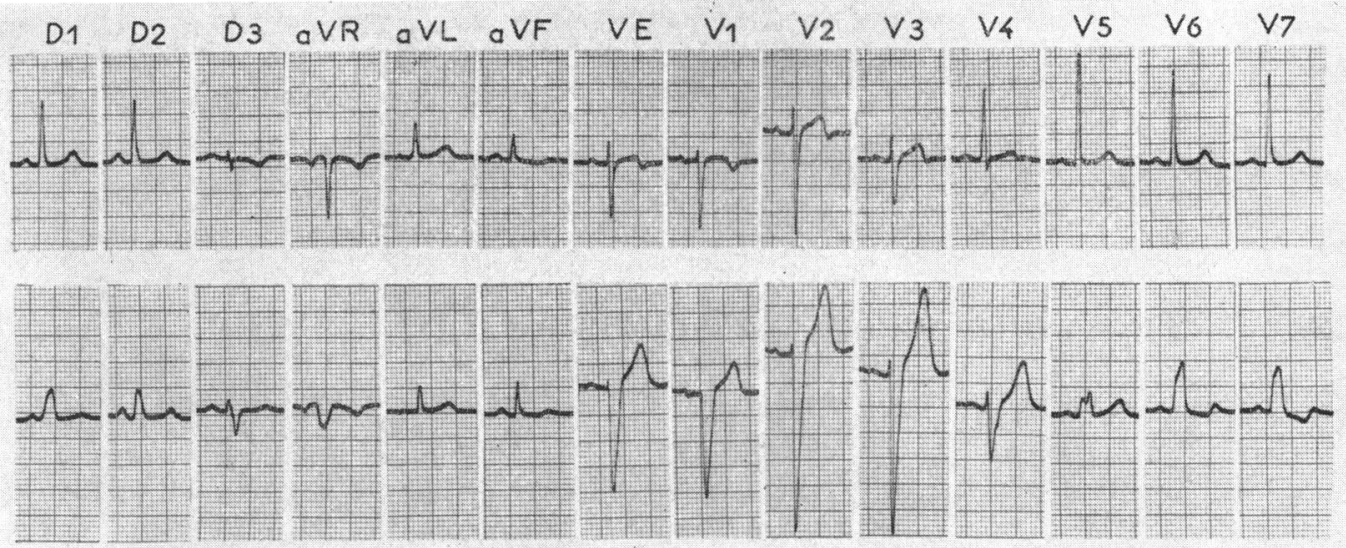

FIG. 2.-Electrocardiogram of the same patient, recorded at different moments of the same session. Top: incomplete left bundle-branch block. Bottom: complete left bundle-branch block.

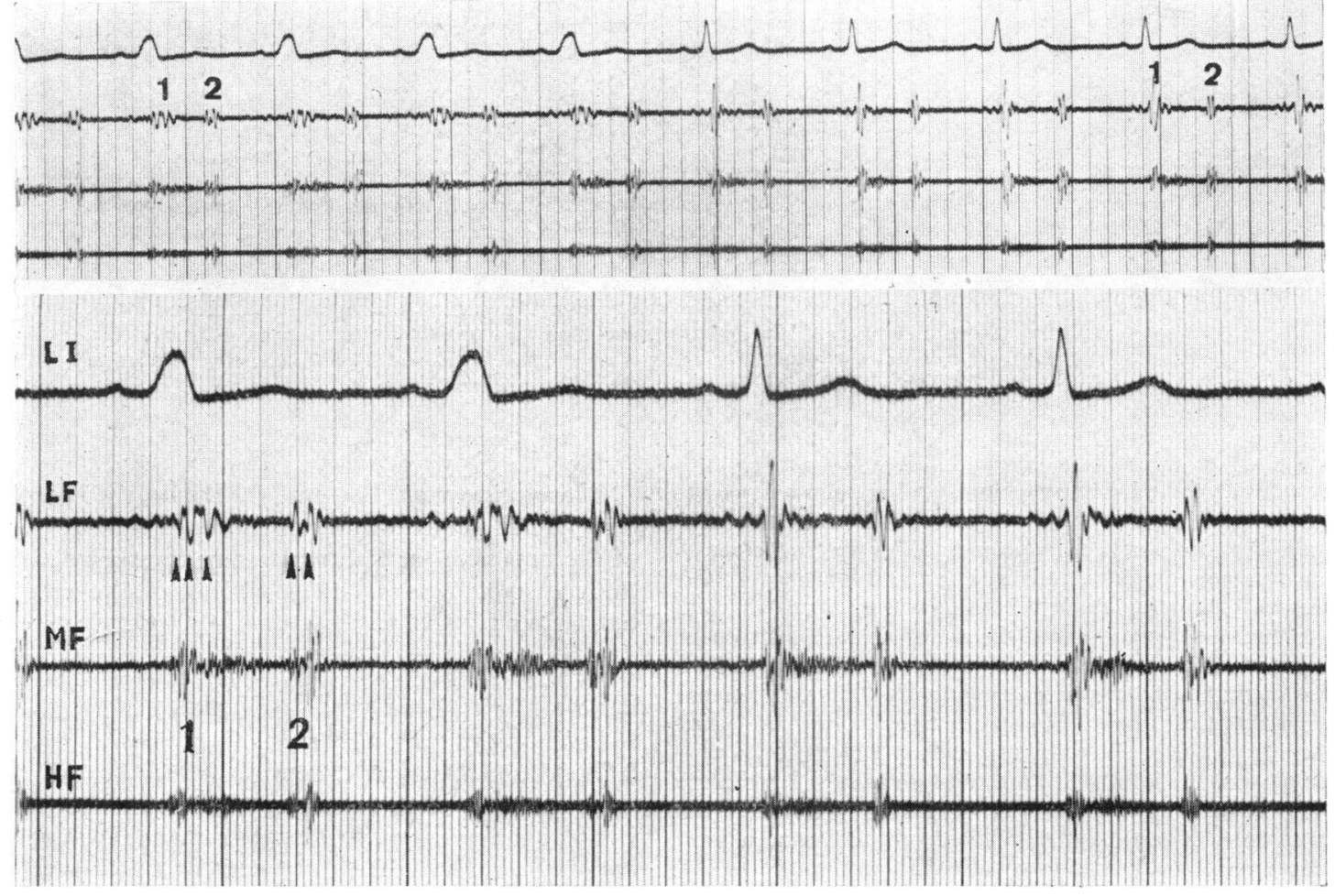

FIG. 3.-Phonocardiogram at the apical area. In both upper and lower parts of the picture, from above downwards: electrocardiographic lead I, low frequency (LF), medium frequency (MF), high frequency (HF). Top: passage from complete to incomplete left bundle-branch block with very slight modification of cardiac rate. Bottom: same picture, enlarged for details. In complete bundle-branch block, the first sound is widened, soft, and dissociated into three discrete waves; the second sound is split. 


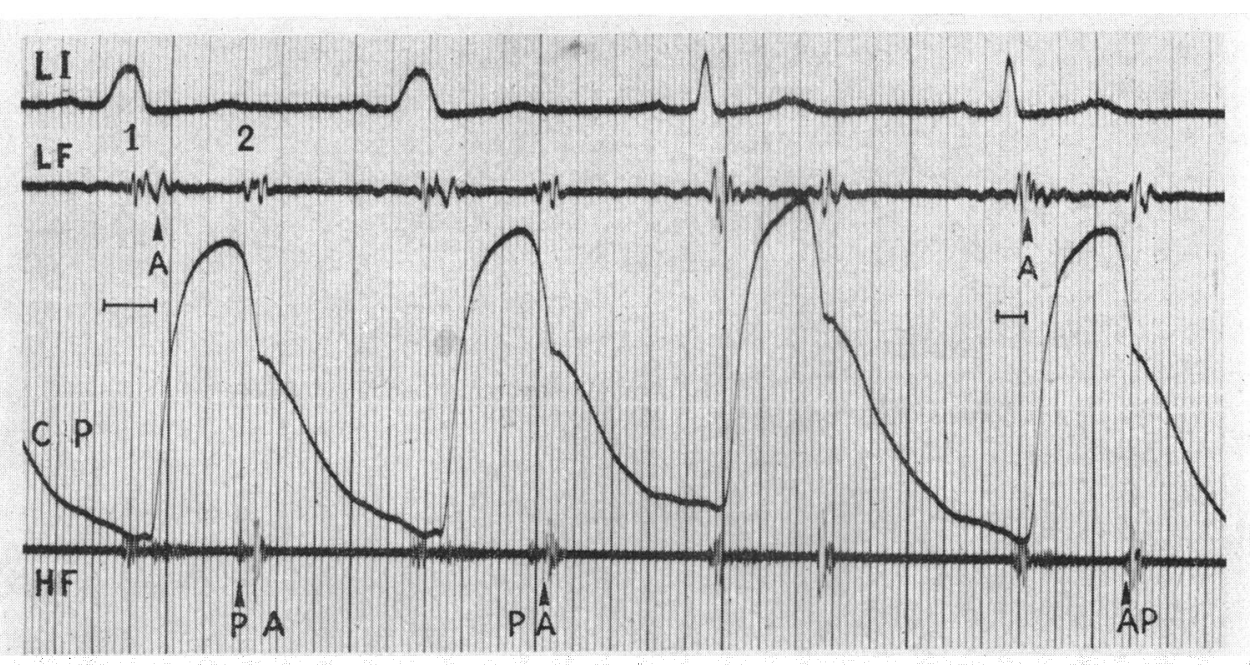

Fig. 4.-Modifications of the heart sounds at the pulmonary area and of the carotid pulse (CP) in complete left bundle-branch block. From above downwards: electrocardiographic lead I, low frequency phonocardiogram (LF), carotid pulse (CP), high frequency phonocardiogram (HF). Note in complete block: (1) the increased interval between the onset of QRS and the onset of CP, shown by $1-1$; (2) the correspondence between the onset of $\mathrm{CP}$ and the third wave of the widened first sound (aortic component of the first sound: upper A); (3) the split second sound (PA), with both components preceding the dicrotic notch of the CP (reversed splitting).

PEC-II, of ventricular contraction, by, respectively, 0.038 and $0.024 \mathrm{sec}$. with a slurring of the onset of PEC-I; (3) the delayed MC notch corresponded then to the second wave of the widened first sound, indicating that mitral closure followed, instead of preceded, tricuspid closure; (4) conspicuously increased isometric relaxation phase, by $0.049 \mathrm{sec}$, with slurring of its terminal portion; and (5) shortening of the ventricular filling phase by 0.098 sec.

\section{Discussion}

In this particular case, the salient modifications following the occurrence of left bundle-branch block were: diminished intensity and increased duration of the first heart sound, with a mitral component following its tricuspid component; reversed splitting of the second heart sound; no delay in onset of left ventricular contraction but delayed left ventricular ejection, due to increased duration of both pre-ejection components; increased isometric

TABLE

COMPARISON BETWEEN TIME INTERVALS OF INCOMPLETE AND COMPLETE LEFT BUNDLE-BRANCH BLOCK

\begin{tabular}{|c|c|c|c|c|c|}
\hline & Intervals & $\begin{array}{l}\text { Incomplete } \\
\text { left bundle- } \\
\text { branch block } \\
\text { (msec.) }\end{array}$ & $\begin{array}{l}\text { Complete } \\
\text { left bundle- } \\
\text { branch block } \\
\text { (msec.) }\end{array}$ & Difference & "p" value \\
\hline Electrocardiogram & $\begin{array}{l}\text { R-R } \\
\text { QRS }\end{array}$ & $\begin{array}{r}803 \\
70\end{array}$ & $\begin{array}{l}758 \\
130\end{array}$ & $\begin{array}{l}-45 \\
+60\end{array}$ & $\begin{array}{l}<0.001 \\
<0.001\end{array}$ \\
\hline Carotid pulse curve & $\underset{\text { EP }}{\text { Q-OCP }}$ & $\begin{array}{r}99 \\
353\end{array}$ & $\begin{array}{l}166 \\
326\end{array}$ & $\begin{array}{l}+67 \\
-27\end{array}$ & $\begin{array}{l}<0.001 \\
\text { N.S. }\end{array}$ \\
\hline Apex cardiogram & $\begin{array}{l}\text { EMI } \\
\text { PEC-I } \\
\text { PEC-II } \\
\text { IR } \\
\text { VF }\end{array}$ & $\begin{array}{r}18 \\
54 \\
27 \\
91 \\
378\end{array}$ & $\begin{array}{r}23 \\
92 \\
51 \\
140 \\
280\end{array}$ & $\begin{array}{l}+5 \\
+38 \\
+24 \\
+49 \\
-98\end{array}$ & $\begin{aligned} & \text { N.S. } \\
&<0.001 \\
&<0.001 \\
&<0.001 \\
&<0.001\end{aligned}$ \\
\hline
\end{tabular}

Note: The numbers are average measurements from 10 cardiac cycles, corrected for heart rate using Bazett's (1920) formula, and expressed in milliseconds. Q-OCP: interval between onset of QRS and onset of CP; EP: ejection period of left ventricular contraction; EMI: electromechanical interval; PEC-I and PEC-II : respectively, first and second pre-ejection components of left ventricular contraction; IR: isometric relaxation phase; VF: ventricular filling phase; NS: not significant. 


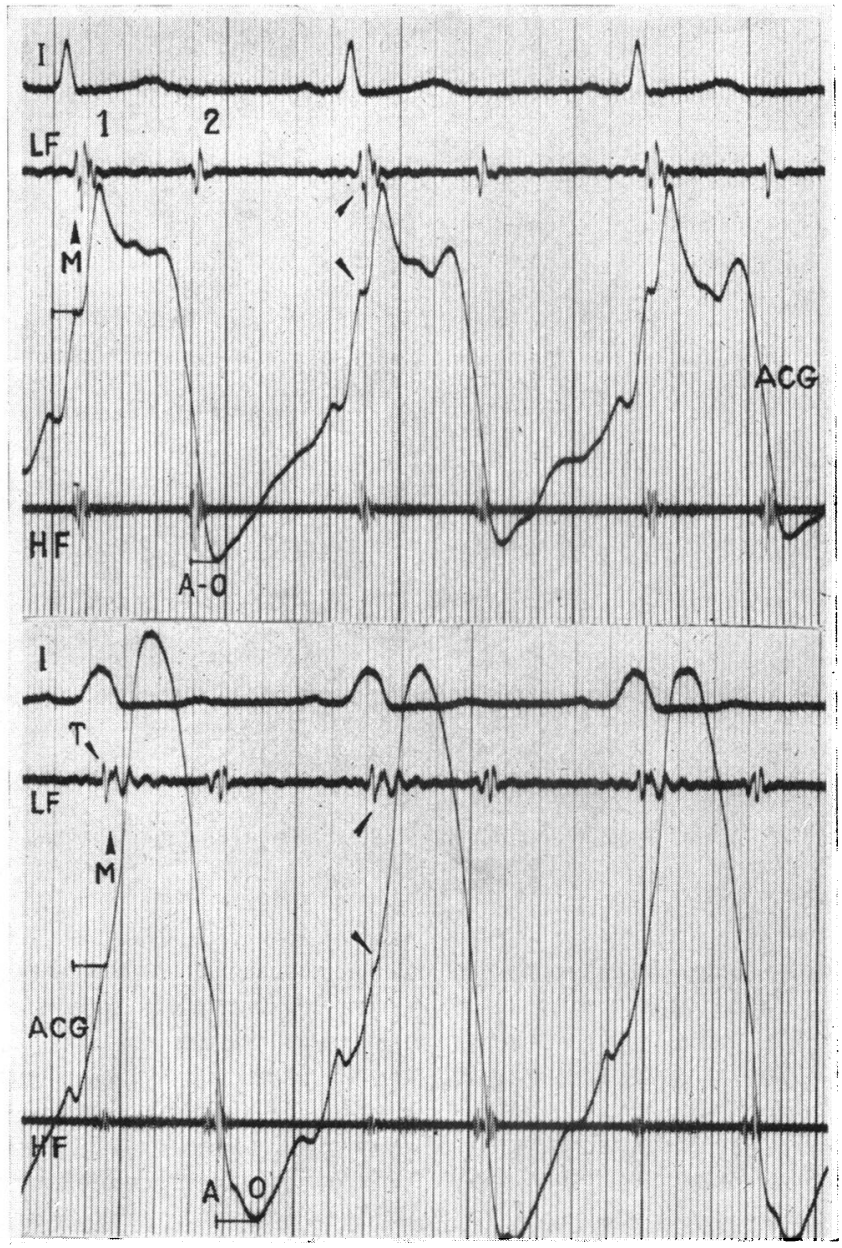

Fig. 5.-Modifications of the heart sounds recorded just inside the apex beat and of the apex cardiogram in complete left bundle-branch block. In both upper and lower halves of the picture, from above downwards: electrocardiographic lead I, low frequency phonocardiogram (LF), apex cardiogram (ACG), high frequency phonocardiogram (HF). Note in complete block (lower half): (1) the delayed mitral valve closure (increase of the interval, marked by the upper 1-, between onset of QRS and the notch on the systolic ascending limb of the ACG); (2) the correspondence between this notch and the second wave (mitral component $M$ ) of the first heart sound, following tricuspid closure $T$; (3) the slurred onset of systolic contraction of the ACG; (4) the increased isometric relaxation phase, from A2 to the point " $O$ " of the ACG (lower I-); (5) the shortening of the ventricular filling phase (between the point " $O$ " and the onset of the following systolic wave of the ACG).

relaxation phase and shortening of left ventricular filling phase.

A soft first heart sound in left bundle-branch block has been known for a long time, but explained differently by various authors (Contro and Luisada, 1952; Leatham, 1954; Bellet, 1963). Haber and Leatham (1965), particularly, because they analysed cases in which left bundle-branch block was associated with severe heart disease, could not ex- clude powerful atrial contraction or a long P-R interval as possible causes of the soft heart sound. In this case, however, it seemed to be due, at least partly, to dissociation of the first heart sound into three of its components, successively tricuspid, then mitral (delayed mitral closure), then aortic (delayed onset of left ventricular ejection).

On the other hand, every author agreed that reversed splitting of the second sound was due to 
delayed closure of the aortic valve. Some attributed this delay to an increased ejection time (Braunwald and Morrow, 1958) but this was not found here, and others attributed it to a delayed onset of left ventricular ejection (Leatham, 1954; Gray, 1956). This we found to be the case, and reversed splitting occurred in spite of slight shortening of systolic ejection time.

Since Wolferth and Margolies (1935) had demonstrated much delayed left ventricular ejection in cases of complete left bundle-branch block, some thought it was due to delayed activation of the left ventricle (Gray, 1956), but most others, using phonocardiographic (Luisada, 1959; Haber and Leatham, 1965), electrokymographic (Segers and Hendrickx, 1951), or hæmodynamic (Braunwald and Morrow, 1957; Bourassa, Boiteau, and Allenstein, 1962) arguments, pointed out that the delay occurred during the contraction itself.

In their recent hæmodynamic study of one case of intermittent left bundle-branch block, Bourassa et al. (1962) found in complete block an undelayed onset of left ventricular contraction but a much prolonged isometric contraction and relaxation, together with an important shortening of the diastolic period. Our findings are almost identical, but because it was possible to localize on the apex cardiogram the moment of mitral closure, the delay could be shown to occur particularly during the first pre-ejection component of ventricular contraction, though its second component was prolonged as well, but to a lesser extent. It may also be worthy of note that the sum total of all increments of the various phases PEC-I, PEC-II, and isometric relaxation $(+0.111 \mathrm{sec}$.) account with reasonable accuracy for the shortening of the ventricular filling phase $(-0.098 \mathrm{sec}$.).

These findings seem to bring some support, at least in this particular case, to the concept of arborization block in "complete left bundle-branch block". Nevertheless they can also be explained on the basis of bundle-branch destruction. Due to the peculiar fan-like anatomy of the left bundlebranch fibres, it has already been shown that, even in cases of typical and permanent electrical patterns of complete left bundle-branch block, the fibres of the left bundle-branch are rarely destroyed completely (Lenègre, 1957). Usually between 10 and 25 per cent of the fibres escape. These intact fibres may be enough to start the left ventricular contraction within normal time limits, but completion of ventricular contraction may depend on impulses spreading across the interventricular septum, from right to left. These impulses travelling through undifferentiated myocardium are variably delayed, resulting in asynchronous contraction and relaxation of the various myocardial fibres, and therefore in a prolonged pre-ejection phase and isometric relaxation phase.

\section{SUMMARY AND CoNCLUSIONS}

This phonocardiographic and mechanocardiographic study of one case of intermittent complete left bundle-branch block, in an otherwise apparently normal heart, showed that complete left bundle-branch block was accompanied by the following modifications: a soft and prolonged first heart sound, with the mitral component following the tricuspid one; reversed splitting of the second heart sound; no delay in the onset of left ventricular contraction; delayed left ventricular ejection due to increased duration of both pre-ejection components of ventricular contraction; an increased isometric relaxation phase; and a shortening of the left ventricular filling phase.

These findings seem to support the mechanism of arborization block, but may also be explained on the basis of left bundle-branch destruction.

We wish to thank Dr. Aubrey Leatham for the advice and help he has given to one of us (J.B.) in the writing of this paper.

\section{REFERENCES}

Bazett, H. C. (1920). An analysis of the time-relations of the electrocardiograms. Heart, 7, 353.

Bellet, S. (1963). Clinical Disorders of the Heart Beat, 2nd ed. Lea \& Febiger, Philadelphia.

Bourassa, M. G., Boiteau, G. M., and Allenstein, B. J. (1962). Hemodynamic studies during intermittent left bundlebranch block. Amer. F. Cardiol., 10, 792.

Braunwald, E., and Morrow, A. G. (1957). Sequence of ventricular contraction in human bundle-branch block; a study based on simultaneous catheterization of both ventricles. Amer. F. Med., 23, 205.

$\longrightarrow$, and - (1958). Origin of heart sounds as elucidated by analysis of the sequence of cardiodynamic events. Circulation, 18, 971.

Contro, S., and Luisada, A. A. (1952). Modifications of the heart sounds in bundle-branch block. F. Mt Sinai Hosp., 19, 70.

Gray, I. R. (1956). Paradoxical splitting of the second heart sound. Brit. Heart f., 18, 21.

Haber, E., and Leatham A. (1965). Splitting of heart sounds from ventricular asynchrony in bundle-branch block, ventricular ectopic beats, and artificial pacing. Brit. Heart F., 27, 691.

Leatham, A. (1954). Splitting of the first and second heart sounds. Lancet, $2,607$.

Lenègre, J. (1957). Contribution à l'étude des blocs de branche comportant notamment les confrontations électriques et histologiques, p. 88. J.-B. Baillière, Paris. [Arch. Mal. Cour, 50, Suppl. 1.]

Luisada, A. A. (1959). Graphic data in bundle-branch block. In Cardiology-An Encyclopadia of the Cardiovascular System, Vol. 3, Part II, p. 87. McGraw-Hill, New York.

Segers, M., and Hendrickx, J. (1951). Étude électrokymographique du délai d'éjection dans les blocs intraventriculaires. Acta cardiol. (Brux.), 6, 150. 
Tafur, E., Cohen, L. S., and Levine, H. D. (1964). The normal apex cardiogram. Its temporal relationship to electrical, acoustic and mechanical cardiac events. Circulation, 30, 381.

Wolferth, C. C., and Margolies, A. (1935). Asynchronism in contraction of the ventricles in the so-called common type of bundle-branch block: Its bearing on the determination of the side of the significant lesion and on the mechanism of split first and second heart sounds. Amer. Heart f., 10, 425. 